\title{
Desafios no incentivo de meninas para a área de Computação
}

\author{
Maria Madalena Gomes de Pontes ${ }^{1}$, Wanessa Paiva Silva ${ }^{1}$, Vanessa F. Dantas ${ }^{1}$, \\ Thaíse K. L. Costa ${ }^{1}$ \\ ${ }^{1}$ Centro de Ciências Aplicadas e Educação - Universidade Federal da Paraíba (UFPB) \\ Caixa Postal: 58297-000 - Rio Tinto - PB - Brasil \\ \{maria.madalena, wanessa.silva, vanessa.dantas, thaise\} @dcx.ufpb.br
}

Abstract. Research has shown that women are strongly underrepresented in computer-related degree programs and occupations. It is important that efforts are made to increase girls' interest in the area and to promote contact with computing resources start from fundamental education. This paper presents the challenges and possibilities experienced by students in Teaching Degree in Computer Science, seeking to encourage girls in technology. Based on the lines of action presented in the article, activities were organized with the purpose of promoting closer ties and cooperation between university and school, as well as creating a support network for female empowerment and training.

Resumo. Na área da Computação mulheres ainda são minorias, por isso é importante promover e manter ações com meninas desde a educação básica, a fim de incentivar o contato dessas com os recursos computacionais, como também despertar interesse pela área e eventualmente impulsionar a busca pela profissão. O presente trabalho apresenta os desafios e possibilidades vivenciadas por licenciandas em Computação na busca de incentivar meninas na tecnologia. Com base em algumas linhas de ações apresentadas no artigo, atividades foram organizadas com o intuito de impulsionar aproximação entre universidade e escola, constituindo uma rede de apoio para empoderamento feminino e oficinas de formação com foco na área de Computação.

\section{Introdução}

O setor de TI (Tecnologia da Informação) é considerado promissor, a revista O Globo (2019) anuncia que "Tecnologia da Informação está em expansão e oferece diversas possibilidades no mercado de trabalho", contudo ainda não há boa diversidade de gênero. De acordo com a Pesquisa Nacional por Amostra de Domicílio (PNAD) do Instituto Brasileiro de Geografia e Estatísticas [IBGE 2009] só 20\% dos profissionais que atuam no mercado de TI são mulheres. Na literatura são encontrados alguns trabalhos que analisam os fatores pelos quais as mulheres não se sentem atraídas por essa área [AIRES et al. 2018] [POSSER e TEIXEIRA 2016] [MARTINS et. al 2019].

Aires et al. (2018) apontam em seu trabalho fatores que, segundo alunas do ensino médio, dificultam o ingresso de mulheres na área de Computação, dentre eles: o machismo; a comparação de sua capacidade com a dos homens; a sensação de isolamento devido a predominância masculina na área; e a ideia imposta de que 
Computação é coisa de homem. Outro fator que dificulta a escolha da profissão é não conhecer o papel das mulheres na Computação, atual e histórico, ou ter pessoas próximas/conhecidas como exemplos inspiradores. Matsu (2017) afirma que a representatividade feminina no setor de TI é primordial tanto para o recrutamento, quanto para a permanência na área, pois ter mulheres como referência e analisar sua trajetória serve como apoio afetivo e inspiração. Diante das premissas expostas, reforçase a importância de incentivo à diversidade de gênero na tecnologia, buscando meios de estimular mulheres na área. Além disso, é essencial promover ações que divulguem a Computação na educação básica a fim de promover o contato entre o educando e os recursos computacionais, com intuito de despertar interesse pela área e eventualmente impulsionar a busca pela profissão. Uma possibilidade para propagação da Computação é a disseminação do pensamento computacional, com o objetivo de desenvolver competências, na qual permite um contato inicial com as possibilidades de atuação da área na tentativa de atrair novos talentos [Santos, Santos e Ellwanger 2017].

Este trabalho tem como objetivo apresentar desafios e possibilidades vivenciadas por licenciandas em Computação na busca de incentivar novas meninas a ingressarem na área de tecnologia. Para isso, o artigo mostra o contexto das alunas de uma escola indígena da cidade de Rio Tinto - PB. Dentre os desafios, destacam-se a falta de perspectiva de continuidade do ensino; baixos recursos da escola e comunidade na qual estão inseridas; e a falta de iniciativas de apoio na escola para incentivo tecnológico e de conhecimento da área de Computação. Como foi identificado na literatura, esses desafios também se fazem presentes em outras realidades, e por isso a importância em discutir ações de enfrentamento como a aproximação universidadeescola, núcleo de apoio e oficinas de formação.

A fim de abordar a temática, o artigo está dividido da seguinte forma: A seção 2 apresenta os desafios na realidade escolar, a seção 3 discute sobre ações de enfrentamento, a seção 4 apresenta as vivências na escola de atuação, a seção 5 contém as considerações finais deste trabalho e ações futuras, e por fim a seção 6 com os agradecimentos.

\section{Desafios na Realidade Escolar}

As alunas da escola indígena da região de Rio Tinto - PB enfrentam vários desafios que precisam ser considerados ao se planejar ações de incentivo à Computação e Tecnologia. Na realidade da comunidade, a primeira questão ao se planejar ações que possam ajudar na formação e mostrar a Computação como espaço de atuação é: Como incentivar meninas que não veem o ensino superior ou técnico como possibilidade de formação profissional?

Percebeu-se que para essas meninas os desafios iam um pouco além, e que deveriam ser tratados em conjunto. É necessário um trabalho paralelo para: mostrar o ensino superior como um caminho de mudança e trabalhar o empoderamento feminino. Dessa forma, foram planejadas três linhas de ações de enfrentamento (seção 3) e executadas atividades (seção 4) com base nessas linhas.

A realidade social e cultural na qual as meninas da região estão inseridas mostram situações como gravidez na adolescência e atribuições de trabalhos domésticos como obrigações adquiridas devido ao gênero. A maioria das alunas não vislumbram ingressar no ensino superior, como também não se enxergam assumindo posições de 
VIII Congresso Brasileiro de Informática na Educação (CBIE 2019)

Anais dos Workshops do VIII Congresso Brasileiro de Informática na Educação (WCBIE 2019)

liderança. Dessa forma, mostra-se a necessidade de promover a capacidade das mulheres, apontando novas possibilidades e incentivando a conquista de outros espaços.

Para o incentivo na área da Computação, diante da realidade que as meninas estão inseridas, se tem a necessidade de desenvolver habilidades de pensamento computacional por meio de formas alternativas que sejam compatíveis com os recursos escolares, com intuito de mostrar a área de Computação como uma oportunidade. Conforme afirma França e Tedesco (2015) "constata-se que os princípios da Computação podem ser ensinados aos estudantes sem depender do uso de uma tecnologia específica". Para isso, essas meninas precisam se sentir capazes enquanto mulheres e intelectualmente, como também conhecer a área para que seja desmistificado que Computação não é para mulher, tendo convicção que podem ser o que quiserem. Nesse contexto, iniciativas de apoio para que meninas persistam nos estudos se tornam indispensáveis.

\section{Ações de Enfrentamento}

As escolas possuem uma diversidade de alunos com realidades e contextos diferentes. Apesar dos avanços tecnológicos, as escolas da rede pública do Brasil são comprometidas por alguns fatores como: infraestrutura, capacitação de docentes, material didático e segurança. Assim como na realidade da escola indígena da região de Rio Tinto - PB, esses desafios também se fazem presentes em outras escolas, e por isso a importância em discutir ações de enfrentamento como a aproximação universidadeescola, núcleo de apoio e oficinas de formação.

\subsection{Aproximação entre Universidade e Escola}

Segundo Gaioso (2005), um dos motivos da evasão universitária é a falta de informações sobre o desenvolvimento da carreira. Dessa forma, a aproximação entre escola e universidade pode auxiliar na desmistificação de cursos e possibilidades profissionais.

Conduzir alunos da escola ao ambiente universitário é importante para que estudantes da educação básica possam conhecer um pouco do futuro espaço de formação, sendo apresentado à infraestrutura da universidade, bem como sua funcionalidade, conhecer o que as pessoas dentro desse universo produzem, seus cursos de graduação e oportunidades de carreira.

Outra alternativa de aproximação é levar ações da universidade para escola, sendo vantajoso para a formação do licenciando, porque tem a oportunidade de conhecer a realidade escolar, planejar e executar ações de intervenção que contribuem não apenas para sua formação enquanto profissional, mas para a realidade escolar na qual essas ações são vivenciadas. De acordo com Santos, Silva e Hinterholz (2017) a integração entre teoria e prática na formação docente é um dos principais desafios, pois evidencia a dificuldade em conseguir associar o conhecimento teórico às suas atividades práticas.

\subsection{Núcleo de apoio}

$\mathrm{O}$ trabalho de empoderamento feminino às adolescentes surge com o intuito de encorajar e despertar o interesse pela busca de habilidades pessoais e profissionais, bem como promover a conscientização acerca do papel individual na luta por uma sociedade 
mais justa. Conforme Magdalena León (1997), para que haja o empoderamento individual, é necessário também incluir as ações coletivas para haver a liberdade/emancipação das dominações. E como empoderá-las? A questão da representatividade feminina é determinante para promover o potencial pessoal em uma sociedade estratificada como o Brasil, visto que mulheres jovens, pobres, negras, quilombolas, indígenas e LGBTs têm menos oportunidades de visibilidade. O trabalho de Farias e Martins (2018) mostra que a invisibilidade feminina começa desde cedo, restringindo as conquistas e possibilidades educacionais das meninas. Algumas figuras femininas e suas contribuições para o desenvolvimento científico foram ocultadas ao longo da história, nesse sentido de acordo com Louzada et al (2019) abordar a importância das mulheres cientistas para motivar meninas e mulheres surge como uma maneira de desmistificar a concepção que apenas homens têm aptidão para a área de exatas, desconstruindo assim concepções preconceituosas. A formação de um núcleo de apoio nas escolas envolvendo a direção escolar, representantes do corpo docente e discente, com apoio da universidade, ajuda com o propósito de estimular à formação de alunas. O núcleo fomenta a promoção de debates sobre empoderamento feminino e planeja ações de ensino e aprendizado na área da Computação e de uso de tecnologias. Essas ações contribuem para a conscientização do papel da mulher na sociedade no conjunto social, cultural e econômico, e para a consolidação de práticas educacionais mais condizentes com a nova realidade que a revolução tecnológica revela.

\subsection{Oficinas de Formação}

Cesário et al. (2017) diz que práticas de incentivo à inserção de mulheres na Computação vêm sendo utilizadas por diversas universidades, empresas e instituições. Nessa linha, a promoção de oficinas de computação para meninas é um tipo de ação realizada em alguns grupos. No trabalho de Amin, Moura, Fernandes, Vargas e Mello (2019) foi abordado uma oficina de conceitos de Computação e algoritmos às meninas de ensino médio o qual foi mostrado exemplos de problemas que poderiam ser resolvidos com mulheres protagonistas da área de Computação como Ada Lovelace e Hedy Lamarr. Já em Moura, Tavares, Mattos e Moreira (2018) foram ministradas oficinas de desenvolvimento de aplicativos utilizando ferramentas computacionais como Lightbot, Code.org, Scratch e MIT App Inventor.

De acordo com França, Silva e Amaral (2013), os cursos de Computação colaboram de modo interdisciplinar, na busca de soluções de problemas diversos através dos princípios do pensamento computacional. O Pensamento Computacional (PC), segundo [Wing 2006] envolve a resolução de problemas, de maneira que um computador (humano ou máquina) pode efetivamente realizar, o que inclui uma gama de ferramentas mentais que refletem a amplitude de natureza transdisciplinar, isto é, não só do campo da Ciência da Computação mas também em outras áreas.

Oficinas de formação sobre pensamento computacional e raciocínio lógico podem ser realizadas no ambiente escolar por meio de atividades desplugadas, como também em plataformas computacionais. Contudo, devido aos obstáculos enfrentados na infraestrutura das escolas brasileiras, principalmente na rede pública, como a falta de formação de professores para os laboratórios, falta de acesso à internet e ausência de suporte e manutenção de equipamentos, as atividades desplugadas são mais utilizadas mediante sua viabilidade. 
VIII Congresso Brasileiro de Informática na Educação (CBIE 2019)

Anais dos Workshops do VIII Congresso Brasileiro de Informática na Educação (WCBIE 2019)

\section{Vivência na Escola}

Em meio às dificuldades como gravidez na adolescência, procura prematura de emprego para ajudar a família, saída da casa dos pais e a falta de incentivo familiar, buscou-se disseminar nas alunas da educação básica uma visão além da escola. Com base nas linhas de ações de enfrentamento, apresentadas na seção 3, licenciandas em Computação organizaram algumas atividades a fim de promover aproximação entre universidade e escola, estímulo a um núcleo de apoio para empoderamento feminino e oficinas de formação com foco na área de Computação. Cada ação foi planejada e executada em momentos diferentes. A ação de visita à universidade e as oficinas possuem roteiros que podem ser reutilizados em outras escolas ${ }^{1,2}$.

Uma das ações realizadas para a aproximação entre escola e universidade foi a visita de meninas do ensino fundamental e médio à UFPB Campus IV ${ }^{1}$ (Figura 1). Nesse momento de imersão das alunas da educação básica em um ambiente universitário, elas tiveram a oportunidade de participar de palestra sobre a contribuição de mulheres para a área de Computação, bem como ouvir relatos de experiência de alunas e ex-alunas de curso da área, buscando mostrar suas dificuldades e superação. $\mathrm{O}$ intuito do momento foi de incentivar as meninas a enxergarem possibilidades de ingresso em um curso superior, apresentando alguns tipos de atividades que a universidade proporciona aos discentes e à comunidade, bem como divulgar os cursos de Exatas com foco nos cursos de tecnologia existentes no Campus. Por meio da visita, as meninas puderam trocar ideias, tirar dúvidas e conhecer um pouco da história e vivência das graduandas e professoras, sendo também apresentadas às instalações universitárias (laboratórios, salas de aula, biblioteca, empresa Júnior, dentre outros).



Figura 1: Visita às instalações da UFPB Campus IV - Rio Tinto.

$\mathrm{Na}$ tentativa de iniciar um núcleo de apoio foi criado um grupo composto por alunas e professores da escola, bem como alunas e professoras da universidade. A proposta do núcleo é, com o apoio das licenciandas em Computação, capacitar alunas

\footnotetext{
${ }^{1}$ Roteiro da visita à universidade - Aproximação entre universidade e escola: $\langle$ https://docs.google.com/document/d/1Ix7vzrOual9kDvT7p-DwBWf1zDYUasf72Hcm0JEwP0A/edit> 2 Roteiros das oficinas: 〈https://docs.google.com/document/d/1pHJCb9kbFkfi3ArvtIKdHD54brVFK0zPERbDkSONzg/edit?usp=sharing $>$
} 
VIII Congresso Brasileiro de Informática na Educação (CBIE 2019)

Anais dos Workshops do VIII Congresso Brasileiro de Informática na Educação (WCBIE 2019)

do núcleo, bem como professores, para que as ações de empoderamento feminino e formação possam ser continuadas. Dessa forma, as oficinas de formação são realizadas em um primeiro momento para integrantes do núcleo, a fim de que estes integrantes adquiram segurança e autonomia para planejamento e execução de novas ações na escola.

Dentre as atividades promovidas por esse núcleo de apoio, tem-se a "mostra de filmes". Nesse tipo de atividade prioriza-se o foco na representação do protagonismo feminino, visando a conscientização e promoção do debate entre as alunas. Na escola foram realizadas sessões com os filmes Estrelas Além do Tempo e Valente. Na mostra do filme Estrelas Além do Tempo, as alunas tiveram a oportunidade de conhecer o papel histórico de mulheres na Computação e o impacto de suas contribuições para a sociedade. No filme Valente, produzido pela Disney/Pixar, alunas debateram sobre implicações que o gênero traz para a vida da personagem principal e suas ações de enfrentamento diante das dificuldades. O objetivo da "mostra de filmes" é inspirar meninas para que se sintam estimuladas a desenvolver seus potenciais, por isso, sempre após a exibição há um momento de discussão e debate sobre o tema que o filme leva a refletir.

Outras ações realizadas foram promoções de oficinas de Computação ${ }^{2}$. Dentre elas: Fantástica Fábrica de Chocolate e Design de Soluções Tecnológicas para Empoderar Mulheres. As oficinas foram aplicadas para um total de 13 participantes do ensino Fundamental e Médio.

A oficina A Fantástica Fábrica de Chocolate foi baseada no artigo de Maciel, Bim e Boscarioli (2012), e consiste na realização de atividades de IHC (Interação Humano-Computador). Durante a oficina, as alunas precisavam realizar tarefas práticas para projetar uma nova fábrica mais fácil para os operários (Oompa-Loompas) trabalharem. As tarefas e conceitos abordados foram: 1) projetar novas portas pelas quais os Oompa-Loompas deviam passar com baldes fumegantes de chocolate líquido (conceito: affordance); 2) projetar um fogão com uma melhor solução para a distribuição dos botões (conceito: mapeamento); 3) planejar um sistema visual de aviso para o controle de uma esteira (conceito: efeito de transferência e estereótipo de usuários); 4) criar uma solução para guardar os utensílios (conceito: restrições visíveis); e 5) criar um painel de controle para os grupos de funções com ícones individuais para cada uma das operações (conceito: design).

As participantes, de modo geral, demonstraram total atenção e interesse nos desafios das tarefas, desenvolvendo boas soluções para os problemas. No início da oficina quando questionadas se conseguiam se visualizar como inventoras ou projetistas, nenhuma se enxergava como tal, já depois da oficina, após verem que eram capazes de propor projetos e soluções para os desafios, conseguiram ver possibilidades na sua capacidade de criação.

Já a oficina de Design de Soluções Tecnológicas para Empoderar Mulheres foi marcada por uma prática envolvendo a prototipagem de solução tecnológica no contexto da comunidade indígena, a qual instigou as participantes a estimularem a criatividade e propor ideias inovadoras que poderiam se transformar em aplicativos para ajudar mulheres nesse contexto particular. (Figura 2)

Nessa oficina foram formadas equipes, sendo três equipes com três meninas e outra com quatro. Cada grupo teve que discutir as ideias (brainstorming) e depois 
VIII Congresso Brasileiro de Informática na Educação (CBIE 2019)

Anais dos Workshops do VIII Congresso Brasileiro de Informática na Educação (WCBIE 2019)

elaborar um cartaz com o protótipo das telas, simulando as funcionalidades do aplicativo. A primeira equipe idealizou o aplicativo Virtue Potiguara com o propósito de divulgar a cultura indígena potiguara. O protótipo continha um quiz e uma seção "Saiba mais" que mostrava textos, imagens e vídeos explicativos.

Outro grupo idealizou a solução tecnológica LookShop, na qual as lojas de roupas, calçados, maquiagens e bijuterias da cidade local (comunidade escolar) disponibilizariam seus produtos. O objetivo da solução proposta pelas alunas era oferecer um aplicativo pelo qual a comunidade pudesse fazer compras a partir da própria residência, recebendo-as em casa, de forma fácil e prática.

O terceiro grupo projetou o aplicativo Mototáxi feminino, que visa a locomoção das mulheres que moram em uma vila indígena próxima à cidade. A ideia do aplicativo é que as mulheres sejam conduzidas apenas por mulheres, a fim de que as passageiras se sintam mais seguras em relação a assédios e abusos do meio de transporte alternativo.

Já a última equipe projetou o aplicativo Sabe Mais, com intuito de auxiliar os alunos nas disciplinas escolares. Nessa proposta, o aplicativo disponibilizaria o assunto de cada aula, vídeos explicativos, exercícios, e outros materiais.

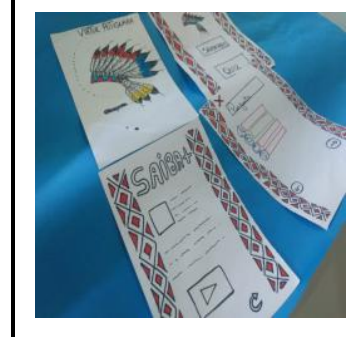

Virtue Potiguara

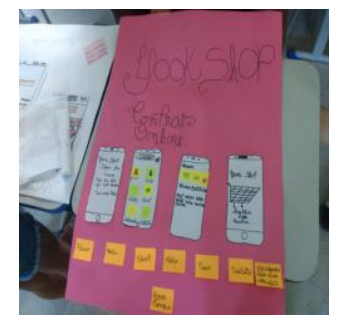

Look Shop



Mototáxi Feminino

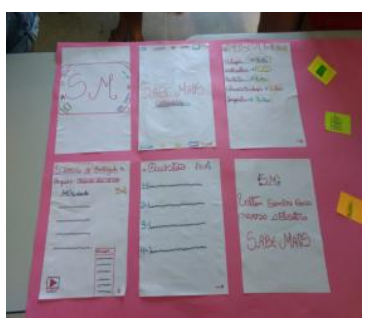

Sabe Mais

Figura 2. Oficina Design de Soluções Tecnológicas para Empoderar Mulheres.

\section{Considerações Finais}

Neste artigo foi apresentado como licenciandas em Computação estão lidando com os desafios na realidade da escola indígena da região de Rio Tinto - PB, buscando incentivar meninas na área de Computação. As ações de enfrentamento abordadas neste trabalho são inspiradas em uma realidade local que, no entanto, se faz presente em outras escolas. Nesse trabalho, as linhas de ações foram planejadas a fim de aproximar a universidade da escola, ajudando na formação de um núcleo de apoio escolar para planejar atividades que auxiliem no empoderamento feminino e formação. Esse trabalho não espera uma mudança momentânea de cenário após a execução de ações pontuais, mas incentiva o núcleo escolar para continuidade e expansão de atividades que possam ajudar meninas a conhecerem a Computação, potencialidades e referências na área, bem como se sentirem capazes, enquanto mulheres, de assumirem diferentes posições na vida.

Dentre as intervenções realizadas, as oficinas desplugadas de Design de Soluções Tecnológicas para Empoderar Mulheres e A Fantástica Fábrica de Chocolate se destacaram por despertar o interesse das alunas em assuntos relacionados à Computação. A motivação das meninas foi refletida na criatividade, aplicação da lógica, 
VIII Congresso Brasileiro de Informática na Educação (CBIE 2019)

Anais dos Workshops do VIII Congresso Brasileiro de Informática na Educação (WCBIE 2019)

trabalho em equipe e posições de liderança, mostrando assim que as ações de enfrentamento são necessárias e servem como uma boa maneira para introduzir os conceitos de computação para meninas que nunca tiveram contato com a área de TI.

Conforme apresentado no trabalho de Mombach et al. (2018) ao realizar uma avaliação através de um questionário sobre a experiência obtida em práticas para criação de aplicativos por meninas do ensino médio, aponta nos dados obtidos que o interesse pela área de Informática obteve um percentual três vezes maior após as meninas terem criado seus próprios aplicativos.

Recomenda-se que práticas sociais e educativas similares às apresentadas neste trabalho sejam desenvolvidas em mais escolas, pois atividades como essas podem aproximar meninas da tecnologia e influenciá-las a optarem por cursos de Computação.

Enquanto licenciandas em Computação, percebemos os desafios de aproximação do meio acadêmico com a escola e a problemática da representatividade feminina na sociedade. Por meio desse trabalho, tivemos a oportunidade de aplicar nossa formação e planejar ações voltadas a uma realidade escolar, considerando dificuldades de infraestrutura e obstáculos sociais. Diante das lições aprendidas como a troca de experiências entre licenciandas e a comunidade, tentativa de contribuição com o processo de tomada de consciência e a sensação de satisfação demonstrada pelas alunas ao decorrer das atividades, é possível concluir que a compreensão e participação do discente vai além da aquisição de conhecimentos técnico-científicos.

Como trabalhos futuros na própria escola, pretende-se dar continuidade às ações para fortalecimento do núcleo de apoio e de formação de meninas. Se faz necessário haver formação das próprias alunas da escola para que elas liderem os núcleos de apoio para as demais meninas, bem como a necessidade de capacitar professores para ajudar nessa aproximação do uso de tecnologias na sala de aula. As ações descritas tem o propósito de incentivar outros licenciandos, professores e pesquisadores no planejamento de intervenções que possam ser realizadas em escolas para que estudantes, em especial meninas, se sintam capazes e encorajados a almejarem continuidade de formação e conhecerem a área da Computação.

\section{Agradecimentos}

Os autores deste trabalho agradecem ao Conselho Nacional de Desenvolvimento Científico e Tecnológico (CNPq) pelo auxílio financeiro e de cotas de IC, ICJ e ATP-A durante a realização das atividades do projeto.

\section{Referências}

Amim, F., Moura, J.; Fernandes, K.; Vargas, K.; Mello, A.(2019). Oficina Gurias na Computação: três horas de imersão feminina na área da Computação discutidas em detalhes. $13^{\circ}$ Women in Information Technology (WIT 2019), volume 13. SBC.

Aires, J., Mattos, G., Oliveira, C., Brito, A., Aragão, A. F., Alves, S., Coelho, T. e Moreira, G. (2018). Barreiras que Impedem a Opção das Meninas pelas Ciências Exatas e Computação: Percepção de Alunas do Ensino Médio. In: Anais do $12^{\circ}$ Women in Information Technology (WIT2018). SBC, 2018. 
VIII Congresso Brasileiro de Informática na Educação (CBIE 2019)

Anais dos Workshops do VIII Congresso Brasileiro de Informática na Educação (WCBIE 2019)

Cesário, G., Silveira, N.G., Bim, S.A., Maciel, C. (2017). "Por Mais Mulheres na Computação: análise dos trabalhos publicados no $\mathrm{X}$ Women in Information Technology". $11^{\circ}$ WIT-Women in Information Technology.

ESTRELAS Além do Tempo. Produção de Theodore Melfi. Estados Unidos:Twentieth Century Fox, 2016. DVD (127 min)

Farias, S., Martins A. (2018). Invisibilidade Feminina e Representações Sociais de Gênero em tecnologia e ciências. Actas do $12^{\circ}$ Congresso Nacional de Psicologia da Saúde.

França, R.; Tedesco, P. (2015). Desafios e oportunidades ao ensino do pensamento computacional na educação básica no Brasil. In Anais dos Workshops do Congresso Brasileiro de Informática na Educação (Vol. 4, p. 1467).

Gaioso, N. P. L. (2005). "O fenômeno da evasão escolar na educação superior no Brasil.” Relatório técnico. Pró-reitoria de Pós-graduação e Pesquisa, Universidade Católica de Brasília.

Instituto Brasileiro de Geografia e Estatística. (2009). "Síntese de indicadores sociais: Uma análise das condições de vida da população brasileira ." In: PNAD ( Pesquisa nacional por Amostras de Domicílio). Disponível em: $<$ http://www.ibge.gov.br/home/estatistica/populacao/trabalhoerendimento/pnad2011/ default.shtm>. Acesso em: 04 de Setembro de 2019.

León, M. (1997). Poder y empoderamiento de las mujeres. Santafé de Bogotá: Tercer Mundo y UN Facultad de Ciencias Humanas.

Louzada, N.; Santana, T.; Assis, I.; Braga, R.; Braga, A. (2019). Agindo sobre a diferença: atividades de empoderamento feminino em prol da permanência de mulheres em cursos de Tecnologia da Informação. In: WOMEN IN INFORMATION TECHNOLOGY (WIT-SBC), 13. , 2019, Belém. Anais do XIII Women in Information Technology. Porto Alegre: Sociedade Brasileira de Computação, july 2019 . p. 69-78.

Martins, A., Silva, J., Santos, J., Rebouças, A. (2019). Fatores que Atraem e Afastam as Meninas de cursos da Área de TI. In: Anais do $13^{\circ}$ Women in Information Technology (WIT 2019). SBC, 2019.

Matsu, C. (2017). Mulheres sempre foram protagonistas em Computação. Disponível em: <https://goo.gl/AJdwBM/>. Acesso em: 03/09/2019.

Mombach, J. G.; Bonfim, C. J. L.; Martins, A. B.N.; Sousa, J. K. L.L. (2018). Práticas de criação de aplicativos móveis como estratégia para o empoderamento feminino. In Anais do Workshop de Informática na Escola, volume 24, p. 687.

Moura, A., Tavares, T., Mattos, G., and Moreira, J. (2018). Incentivando alunas do ensino médio a ingressarem em carreiras de ciência e tecnologia na paraíba. In $12^{\circ}$ Women in Information Technology (WIT 2018), volume 12. SBC.

Oliveira, L.; Castelini, P.; Silva, C.A.; Amaral, M.A. ( "Oficina de Computação Desplugada - IHC: "A fantástica fábrica de chocolate". Disponível em: $<$ https://rive.google.com/open?id=1 faBuCDRi1Hlobo8hvYko8nRWaBhsnDfe $>$. Acesso em 03/09/2019 
VIII Congresso Brasileiro de Informática na Educação (CBIE 2019)

Anais dos Workshops do VIII Congresso Brasileiro de Informática na Educação (WCBIE 2019)

O Globo. (2019). Como escolher um curso na promissora área de TI? Disponível em: $<$ https://oglobo.globo.com/sociedade/educacao/enem-e-vestibular/como-escolherum-curso-na-promissora-area-de-ti-23488249/> . Acesso em: 06/09/2019.

Posser, C. V.; Teixeira, A. C. (2016). Mulheres que aprendem informática: Um estudo de gênero na área de TI. Anais do Workshop de Informática na Escola. 2016. p. 707.

Santos, M. P.; Santos, C. P.; Ellwanger, C.(2017). A Computação sob a Ótica de Meninas do Ensino Médio. In: Anais do XI Women in Information Technology (WIT 2017). SBC, 2017.

Santos, W. O.; Silva, C.; Hinterholz, L. (2017). Licenciatura em computação: Desafios e oportunidades na perspectiva do estudante. In Anais do Workshop de Informática na Escola (Vol. 23, No. 1, p. 885).

VALENTE. direção de Mark Andrews, Brenda Chapman e Steve Purcell. Estados Unidos: Pixar, 2012. DVD (2h 07min)

Wing, J. M. (2006). Computational thinking. Communications of the ACM. 